\title{
X-RAY AND EUV SPECTRA OF SOLAR FLARES AND LABORATORY PLASMAS
}

\author{
G. A. DOSCHEK \\ E. O. Hulburt Center for Space Research, Naval Research Laboratory, \\ Washington, D.C. 20375, U.S.A.
}

\begin{abstract}
Recent laboratory work relevant to solar flares on the spectroscopy of highly ionized atoms is reviewed. Much of this work has concerned the X-ray and EUV spectrum of iron ions, Fe xVIIIFe XXIV, which produce prominent emission lines in the spectra of solar flares. Also discussed are recently obtained laboratory X-ray spectra of emission lines of hydrogen-like and helium-like ions, and associated satellite lines due to transitions of the type, $1 s 2 l-2 p 2 l, 1 s^{2} 2 l-1 s 2 p 2 l$, and $1 s^{2} 2 l-$ $-1 s 2 l 3 p$. Satellite lines have also been identified in spectra of solar flares, and can be used to determine the electron temperature of the plasma. The laboratory work is important in the planning of future experiments in solar flare X-ray and EUV spectroscopy.
\end{abstract}

\section{Introduction}

During the last decade, a considerable amount of phenomenological information has been obtained on the X-ray and EUV radiations emitted by solar flare plasmas. This extension of our knowledge of flares to the very short wavelengths is a product of unmanned and manned space vehicles; the most recent highlight being the successful Skylab Mission. X-ray crystal spectrometers and broadband X-ray detectors aboard unmanned satellites such as the Orbiting Solar Observatories (OSO) and the Solrad satellites, have revealed the existence of high electron temperature $\left(T_{\mathrm{e}} \approx 20 \times 10^{6} \mathrm{~K}\right)$ plasmas associated with solar flares, that emit intense line and continuum radiation in the X-ray region below $100 \AA$. These plasmas appear to be largely thermal in nature; in contrast to the hard X-ray emission at energies greater than about $7 \mathrm{keV}$, which appears to have a non-thermal origin (Kane, 1974). The Soft X-ray plasmas are temporally associated with the $\mathrm{H} \alpha$ event, and recent NRL and AS \& E Skylab observations indicate that these plasmas are confined to loop-like structures (Widing, 1975; Krieger et al., 1975).

This review will consider some of the details of the soft X-ray emission line spectrum, and in particular will compare the solar flare spectra with recently obtained laboratory spectra of similar high temperature plasmas. The results of the laboratory work make possible future solar flare X-ray and EUV observations that offer considerable promise for determining the physical conditions in high temperature flare plasmas. This information is important for testing various models of solar flares.

\section{Flare Spectra}

At electron temperatures of $20 \times 10^{6} \mathrm{~K}$, most of the abundant solar elements are ionized to the hydrogen-like and helium-like ionization stages. The emission line spectra of the elements carbon through calcium consist primarily of the resonance 
lines and principal series lines of the hydrogen-like and helium-like ions. These lines fall between $\sim 2.7 \AA$ and $\sim 41 \AA$, and are produced primarily by electron impact excitation of ions in the ground state.

Iron is the only appreciably abundant solar element that is significantly heavier than the other elements. The relatively larger ionization potential of iron ions insures that iron ions other than hydrogen-like and helium-like ions will also be present in high temperature plasmas near $10 \times 10^{6} \mathrm{~K}$. This is indeed observed in flare spectra, and lines from iron ionization stages from Fe XVII through Fe XXV are prominent. In contrast to flares, only the Fe XVII lines are strong in the X-ray spectra of active regions.

The intense flare iron lines emit in three relatively narrow spectral ranges. The resonance transitions $2 s^{2} 2 p^{k}-2 s 2 p^{k+1}$ fall in the EUV region from $\sim 80 \AA$ to $\sim 260 \AA$. Actually, only the two Fe xxIv lines $\left({ }^{2} S-{ }^{2} P\right)$ fall near the long wavelength end of this region (192 $\AA$ and $256 \AA$ ). Most of the other iron lines from Fe XVIII through Fe XXIII are confined between $\sim 80 \AA$ and $\sim 150 \AA$.

Iron lines from $\mathrm{Fe}$ XVII through Fe XXIV involving $\Delta n \geqslant 1$ type transitions fall between $\sim 7.0 \AA$ and $17 \AA$. These lines are due to transitions of the type, $2 s^{2} 2 p^{k}-$ $-2 s^{2} 2 p^{k-1} n l$, and were first observed in flare spectra by Neupert et al. (1967). The $n d$ transitions are predicted to be considerably stronger than the $n s$ transitions (Fawcett et al., 1974).

Finally, a prominent spectral feature has been observed between $1.85 \AA$ and $1.93 \AA$ in solar flare spectra (Neupert et al., 1967; Meekins et al., 1968, 1970; Doschek et al., 1971; Grineva et al., 1973). This feature is due to emission from helium-like Fe xxv, and innershell emission from lower iron ionization stages, i.e., $1 s^{2} 2 s^{2} 2 p^{k}-1 s 2 s^{2} 2 p^{k+1}$. Most of the innershell emission is due to lines of lithium-like Fe xxIV. All three of these iron-line groupings are important for investigating high temperature plasmas.

The innershell transitions, or satellite lines, have also been observed for elements lighter than iron. Most of these lines have been identified as transitions in helium-like and lithium-like ions (Gabriel and Jordan, 1969a; Walker and Rugge, 1971; Neupert, 1971; Doschek, 1972; Feldman et al., 1974a). The wavelengths of most of the satellite lines fall close to the wavelengths of the resonance lines of the hydrogen-like and helium-like ions, and are stronger relative to the resonance lines in spectra of the heavier elements. Satellite lines due to transitions in beryllium-like ions and lower ionization stages have not been conclusively identified for any element other than iron.

Most of the available X-ray flare spectra have been obtained from groups at the Naval Research Laboratory (OSO 4, 6), Goddard Space Flight Center (OSO 3, 5) and at the Lebedev Institute in Moscow (Mandelstam and his colleagues). Neupert and his colleagues at Goddard are the only group to have obtained high resolution EUV iron-line spectra of flares near $100 \AA$.

Figures 1-3 show X-ray and EUV spectra from several large flares. These spectra illustrate the major spectral features discussed above. In addition, Figures 2 and 3 show active region and quiet Sun spectra for comparison with the flare spectrum. Emission of iron ions from Fe XVIII through Fe XXIV is either weak or absent in the active region and quiet Sun spectra, but strong during flares. 

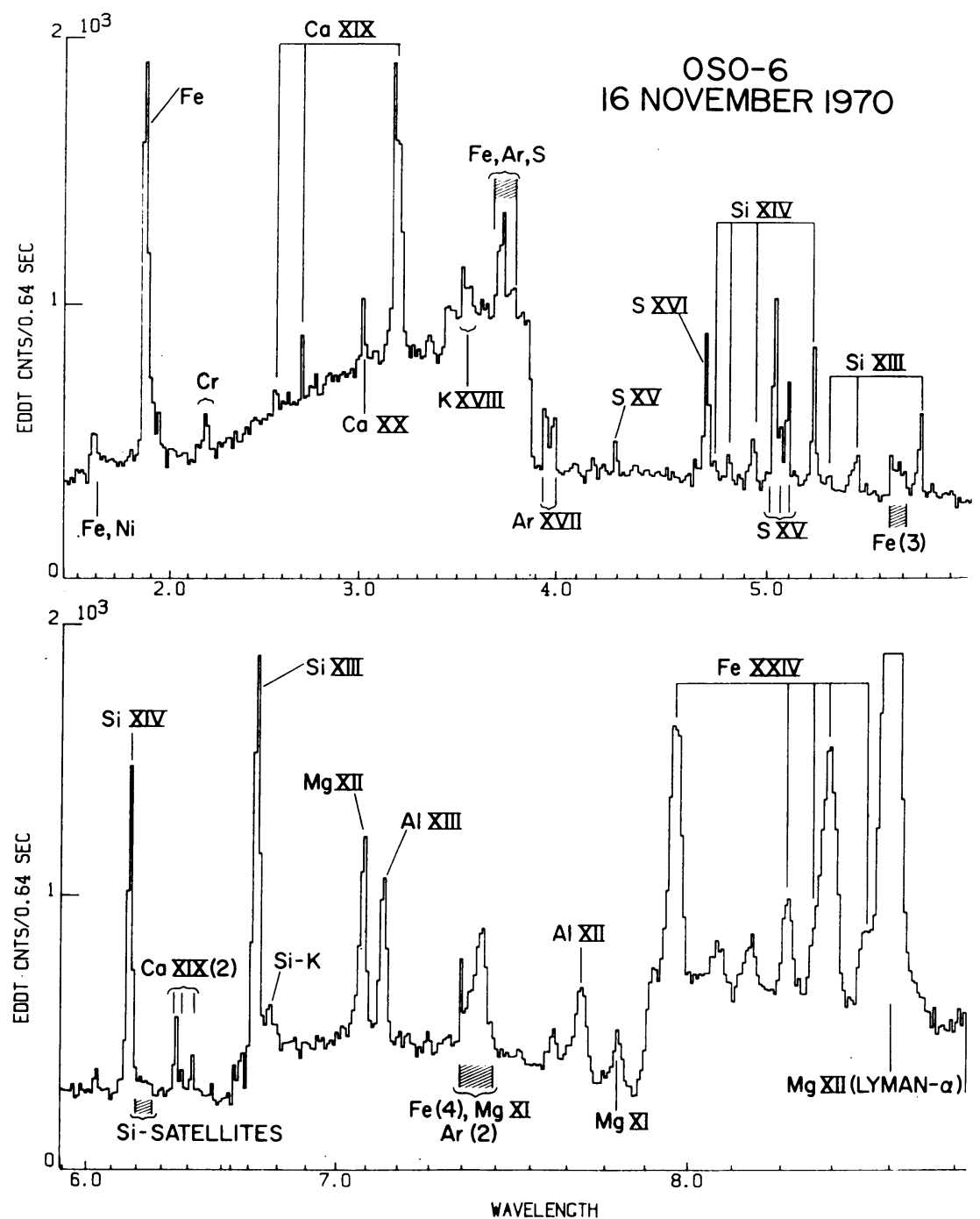

Fig. 1. A solar flare spectrum obtained from an NRL spectrometer on OSO 6. The spectrum is dominated by emission from the resonance lines of hydrogen-like and helium-like ions. Lines of Fe xxIv $\left(2 l-4 l^{\prime}\right)$ are also prominent. The edges in the continuum are instrumental.

In Figures 1 and 2 the emission from the strongest lithium-like satellite lines occurs close to, and on the long wavelength side of the helium-like resonance lines, and is blended with the intercombination $\left(1 s^{2}{ }^{1} S_{0}-1 s 2 p^{3} P_{1,2}\right)$ and forbidden lines $\left(1 s^{2}{ }^{1} S_{0}-1 s 2 s^{3} S_{1}\right)$ of the helium-like ions. A detailed list of flare emission lines and identifications for the wavelength region from $\sim 1 \AA$ to $8.5 \AA$ that summarizes the observations up to 1971 has been published by Doschek (1972). Since then Doschek et al. (1973) and Neupert et al. (1973) have also published wavelengths and identifications for flare spectra in the region from $8.5 \AA$ to $21 \AA$. Wavelengths and identifica- 

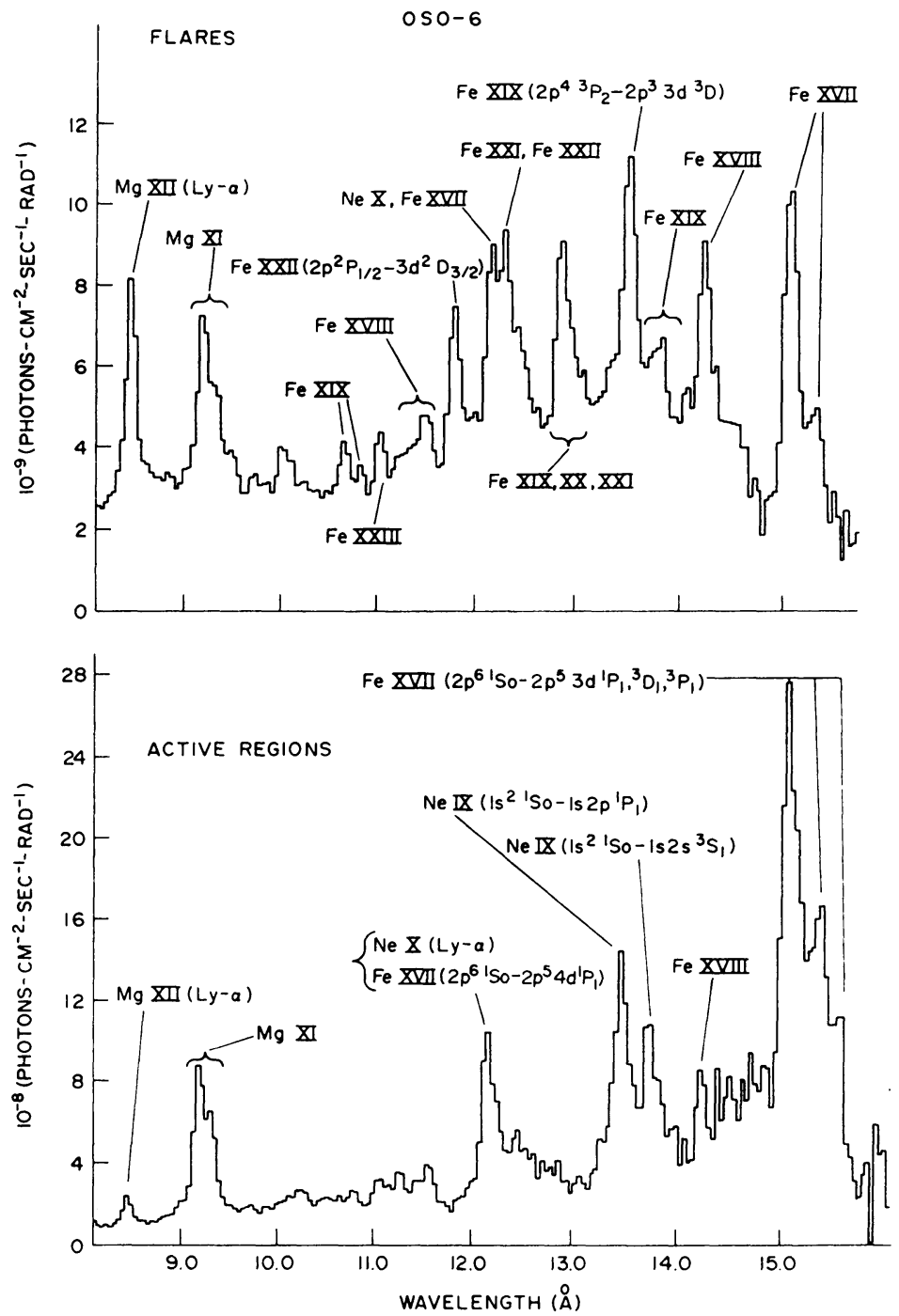

Fig. 2. Solar flare and active region spectra obtained from an NRL spectrometer on OSO 6. Emission from Fe XVIII-Fe XxIV is strong in the flare spectrum but weak or absent in the active region spectrum.

tions for the flare lines near $100 \AA$ (Figure 3 ) have been given recently by Kastner et al. (1974). The $100 \AA$ lines have also been recently observed in laboratory spectra of considerably higher resolution by Feldman et al. (1973a, 1974b) and Doschek et al. $(1974 \mathrm{a}, \mathrm{b})$, and detailed classifications have been published. These results will be discussed further below.

Almost all of the existing flare spectra have low resolution compared with the resolution that is needed to use the full diagnostic capabilities of the spectrum. This will 


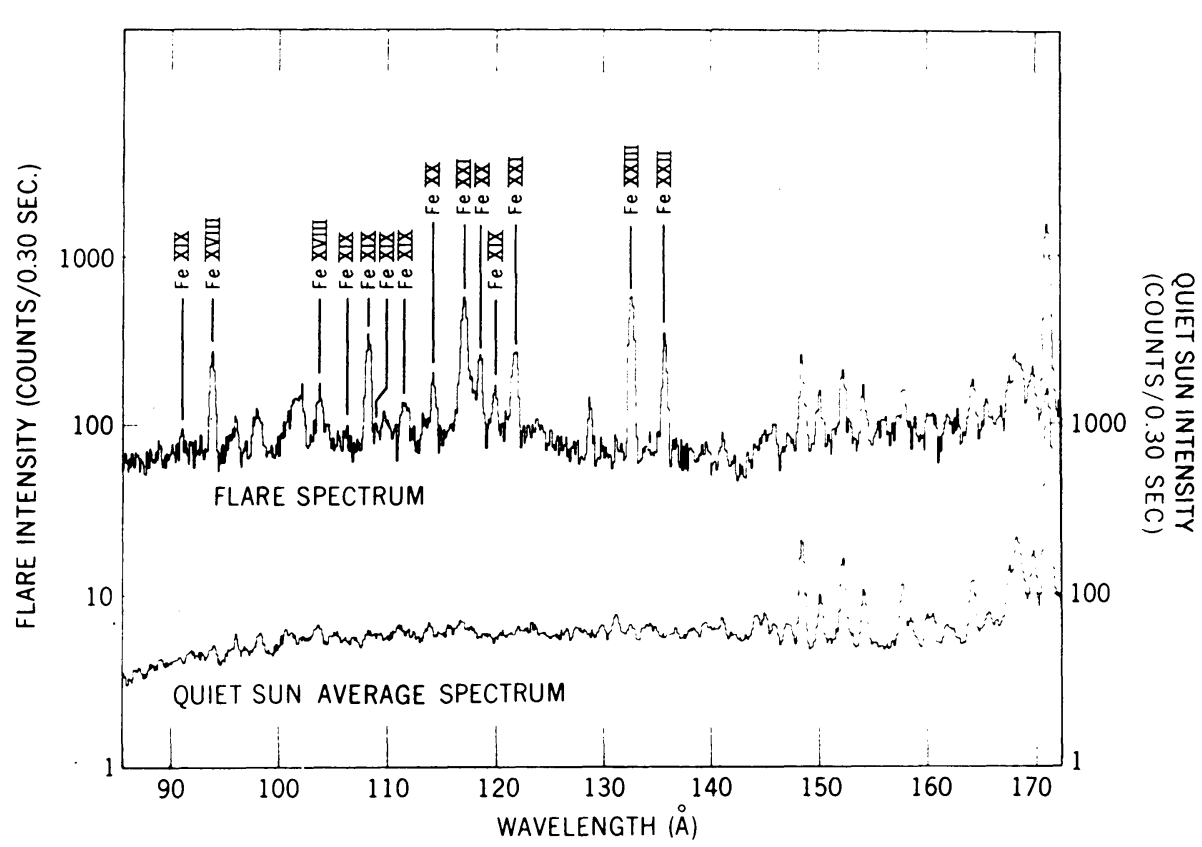

Fig. 3. Solar flare and quiet Sun spectra obtained from a GSFC spectrometer on OSO 5. Emission from Fe XVIII-Fe XXIII is strong in the flare spectrum. Some of the identifications are incorrect. (Spectrum courtesy of S. O. Kastner and W. M. Neupert.)

become clear upon inspecting typical laboratory spectra to be shown below. The majority of the existing solar spectra were obtained by experiments that sacrificed spectral resolution for a large total wavelength coverage with reasonable temporal resolution. The subsequent analysis of the solar data and the analysis of the laboratory data have shown that the full diagnostic possibilities of the spectrum may be exploited by employing much higher wavelength resolution and much higher temporal resolution, over narrow selected wavelength bands.

\section{Recent Results from Laboratory Plasmas}

\section{(a) LABORATORY AND SOLAR PLASMAS}

Generating high temperature laboratory plasmas is motivated by the thermonuclear fusion program and the desire to support astrophysical programs. Laboratory sources that are capable of producing highly ionized atoms include the low inductance vacuum spark, thetapinch sources, Tokamak-type magnetic traps, plasma focus machines, and the focussed pulse of a high power laser. These devices are described in detail in the literature, e.g., Elton and Lee (1972), Burgess (1972), and Fawcett (1973). The low inductance spark devices described by Cohen et al. (1968) at Goddard, Lee and Elton (1971) at NRL, and Schwob and Fraenkel (1972) at the Hebrew University are capable of producing and exciting Fe Xxv and Fe xxvI. 
Recently, high power lasers have been used to generate high temperature plasmas. A high power laser pulse is focused onto a solid target of the material under investigation. Typically, the NRL glass laser can deliver about $60 \mathrm{~J}$ onto a target in $0.9 \mathrm{~ns}$. This energy is converted into thermal energy of the target material and a high temperature plasma is formed that expands outward from the target. The dense regions of the NRL plasmas are about $100 \mu \mathrm{m}$ in size, and exist on the order of nanoseconds.

Although there are fundamental differences between laboratory and flare plasmas, e.g., the electron densities of vacuum spark and laser-produced plasmas are $\approx 10^{19} \mathrm{~cm}^{-3}-10^{21} \mathrm{~cm}^{-3}$ (Lee and Elton, 1971; Feldman et al., 1974a), the similarities between flare and laboratory plasmas are useful and significant. The electron temperatures, or excitation conditions if thermal equilibrium is not assumed, are nearly the same in laboratory and flare plasmas. Therefore many of the lines that are observed in solar plasmas are also observed in the laboratory. The additional permitted lines that are strong in laboratory spectra due to the statistical populating of levels near the ground state are an advantage in making line identifications.

Another significant manner in which laboratory plasmas resemble solar flare plasmas is in the overall phenomenological appearance of the EUV and X-ray radiations from both types of plasmas (Elton and Lee, 1972). There is a remarkable similarity regarding the sequence of emission of hard X-rays, microwaves, and soft $\mathrm{X}$-rays. There is also a close similarity in the time-flux profiles of the various types of radiations. These points have been emphasized most recently by Lee (1974), and Figure 4 shows a typical hard X-ray spectrum of a low inductance vacuum spark plasma. As with solar flare plasmas, a combination thermal spectrum and nonthermal power law spectrum can be fitted to the data.

\section{(b) IRON LINES IN LABORATORY AND FLARE SPECTRA}

The spectra shown in Figures 1-3 indicate the importance of iron line emission in solar flare spectra. Line emission from a large number of iron ionization stages offers the possibility of treating the iron line fluxes in terms of a Pottasch-type analysis, i.e., each ion is assumed to emit at its temperature of maximum emitting efficiency. Simultaneous observations of iron lines from different ionization stages with collimated instruments will enable the emission measure-temperature distribution to be determined as a function of position and time over the flare area. A similar analysis using the resonance lines of hydrogen-like, helium-like, and lithium-like ions alone is more difficult because of the high temperature tails of their excitation functions (Jordan, 1969, 1970).

Iron-line emission near $1.85 \AA$ will be discussed in the next section. In this section iron-line emission from $\sim 7 \AA$ to $17 \AA$ and line emission near $100 \AA$ are discussed.

The solar flare spectra obtained to date do not have sufficient resolution to resolve the blends of iron lines due to the transitions, $2 s^{2} 2 p^{k}-2 s^{2} 2 p^{k-1} n l, n \geqslant 1$, in the $\sim 7 \AA$ to $17 \AA$ region. Laboratory spectra of iron in this wavelength range are quite complex (Feldman and Cohen, 1968; Cohen and Feldman, 1970; Connerade et al., 1970). There is considerable overlapping of lines of different ionization stages. The complexity of 


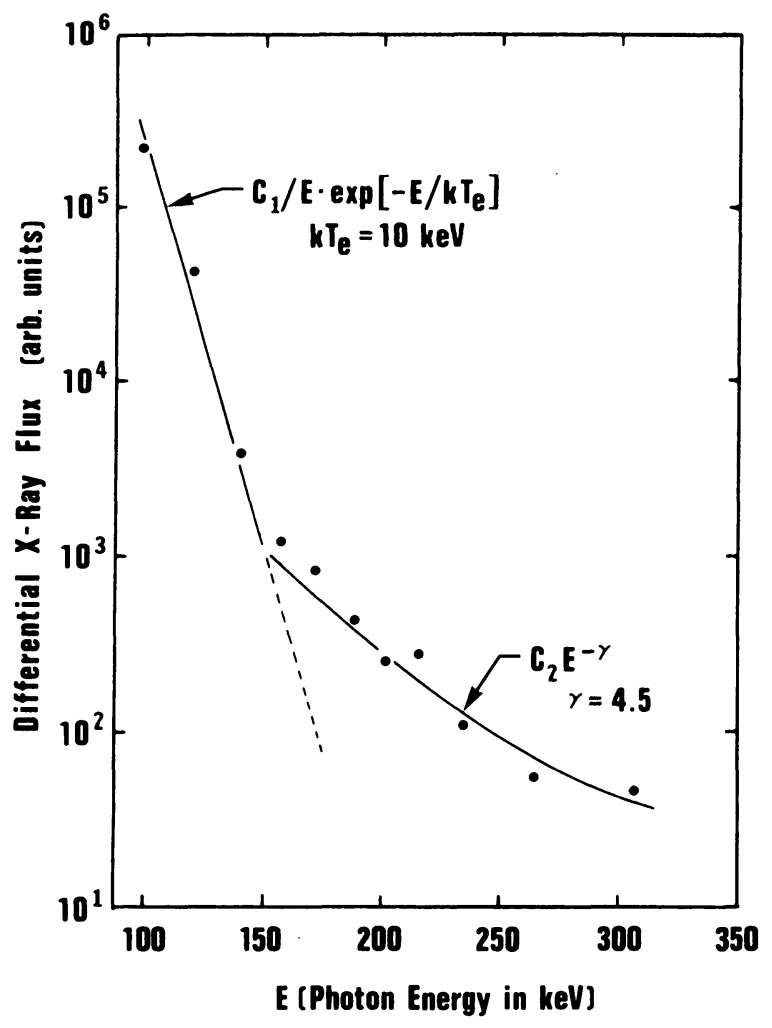

Fig. 4. Hard X-ray spectrum of a vacuum spark plasma. Both a thermal and non-thermal spectrum are combined to fit the data. (Courtesy of T. N. Lee.)

the laboratory spectra is also due in part to statistical populating of the low lying levels of the $2 s^{2} 2 p^{k}$ and $2 s 2 p^{k+1}$ configurations at the high electron densities in laboratory plasmas, which leads to a large number of lines in the laboratory spectrum.

Considerable work on the laboratory spectra is still necessary. However, some recent progress has been reported. Feldman et al. (1973b) have identified or reclassified 27 lines of Fe XVIII between $13.95 \AA$ and $16.27 \AA$, and Fawcett et al.(1974a) have recently discussed the expected solar flare spectrum of iron due to Fe XIX through Fe XXIV. Fawcett et al. point out that the solar flare spectrum should be considerably simpler than the laboratory spectrum, because most of the iron ions are in the ground state. Doschek et al. $(1972,1973)$ have identified lines of Fe XXIII and Fe XXIV near $8 \AA$ in flare spectra, and have shown that the Fe xxIv lines near $10.6 \AA(2 s-3 p)$ are blended (at the low resolution of the available flare spectra) with lines that are probably due to $\mathrm{Fe} \operatorname{xIX}(2 p-4 d)$.

Most of the recent line identifications of highly ionized iron have relied in part on ab initio theoretical wavelength calculations, as well as on the extrapolation of identified lines of lighter elements along the iso-electronic sequences. Many of the recent theoretical calculations have been done by R. D. Cowan at the Los Alamos Scientific 
Laboratory. Cowan's ab initio wavelengths are accurate to about $\pm 0.03 \AA$ at $X$-ray wavelengths, and to about $\pm 1-5 \AA$ for iron lines due to $2 s^{2} 2 p^{k}-2 s 2 p^{k+1}$ transitions near $100 \AA$.

Recently, there has been considerable progress in identifying the iron lines near $100 \AA$ (Feldman et al., 1973a, 1974b; and Doschek et al., 1974a, Fawcett et al., 1974b). Most of this work is based on the analysis of laboratory spectra obtained at NRL from laser-produced plasmas. Kastner et al. (1974) have also recently identified some of these lines in flare spectra obtained by Goddard instruments on OSO 5. Their identifications are based on some of the laboratory work, and on iso-electronic extrapolations. The low resolution of the flare spectra make the line identifications difficult.

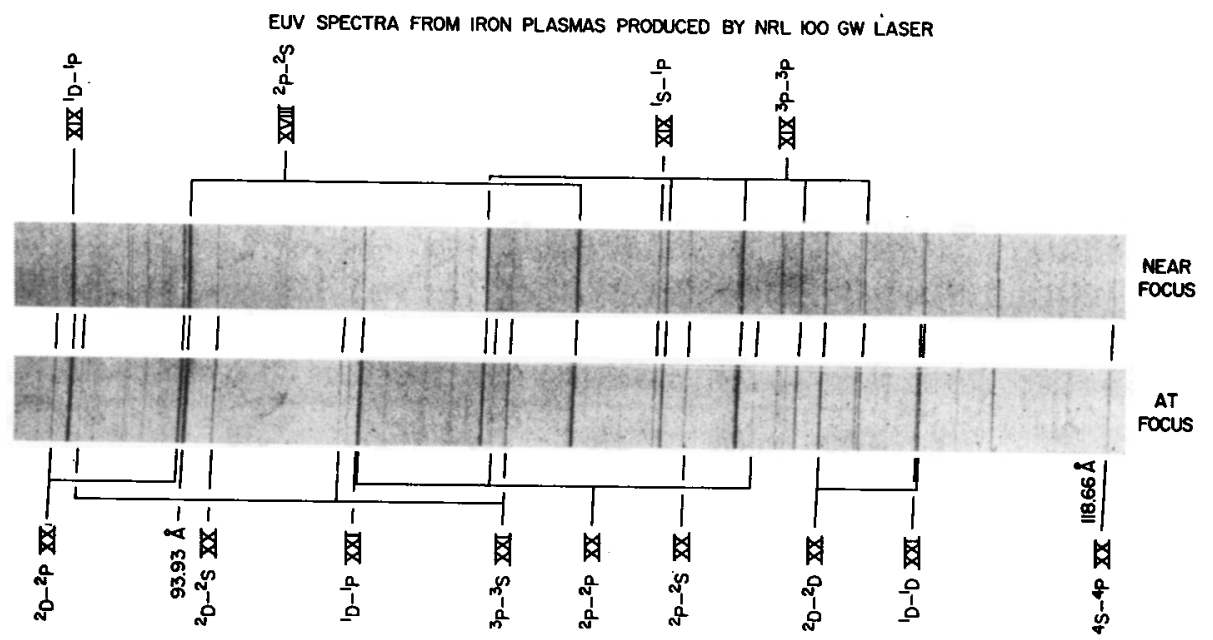

Fig. 5. Iron-line spectra of laser-produced plasmas. Lines of Fe Xx and Fe XXI are substantially stronger in the 'at focus' spectrum than in the 'near focus' spectrum.

Figure 5 shows iron line spectra obtained from NRL $100 \mathrm{GW}$ laser-produced plasmas. By changing the position of the focusing lens (see p. 170), the excitation conditions of the plasma could be varied. The higher ionization stage lines are stronger in the spectrum obtained with the lens well-focused. In this manner, lines of Fe XIX, Fe XX, and Fe XXI can be distinguished from one another by visual inspection.

The detailed identifications of the lines in Figure 5 relied on the earlier work on these iso-electronic sequences given in Moore's Atomic Energy Levels, and on the extension of these data to elements up through $\sim$ vanadium by Fawcett and colleagues at Culham. A summary of the data before the recent work at NRL is given by Fawcett (1971), and Figure 6 shows three spectra of titanium, scandium, and vanadium obtained by Fawcett. The work at NRL confirms most of the identifications of the $2 s^{2} 2 p^{k}-2 s 2 p^{k+1}$ lines of the fluorine, oxygen, nitrogen, and carbon iso-electronic sequences in these lighter elements, and in some cases extends the identifications up through nickel. 


\section{SPECTRA RESULTING FROM TRANSITIONS BETWEEN GROUND TERMS IN SCANDIUM, TITANIUM AND VANADIUM}

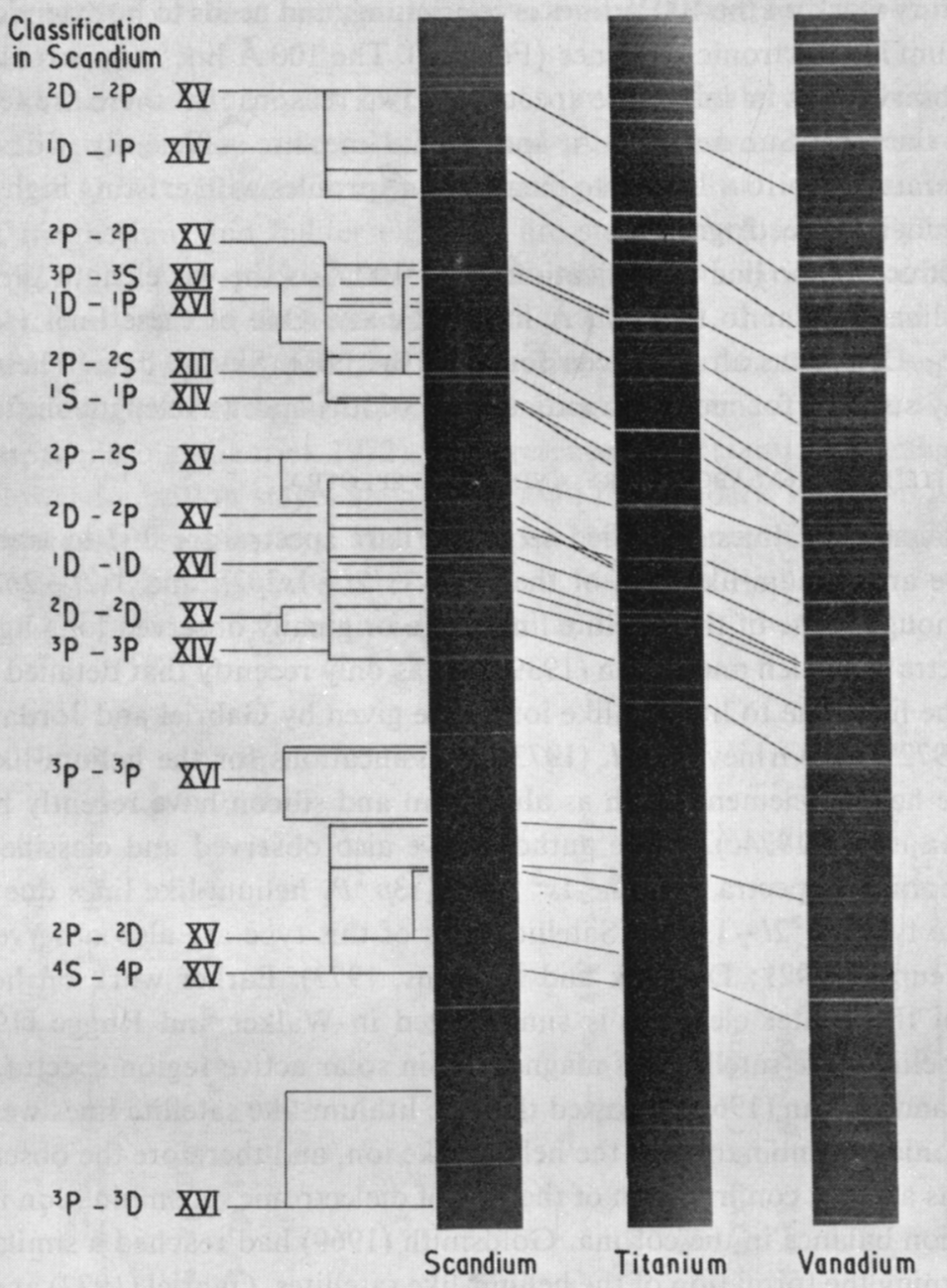

Fig. 6. Laboratory spectra showing lines due to transitions of the type $2 s^{2} 2 p^{k}-2 s 2 p^{k+1}$, in ions of the elements, titanium, scandium, and vanadium. Identifications of lines in these elements is important for identifying the same transitions in iron and nickel. (Spectra courtesy of B. C. Fawcett.)

It is interesting to compare the laboratory iron spectrum of Figure 5 with the solar flare spectrum shown in Figure 3. Because electron densities of flare plasmas $\left(\approx 5 \times 10^{10} \mathrm{~cm}^{-3}\right.$, Cowan and Widing, 1973) are orders of magnitude less than the densities of laboratory plasmas, some of the lines that are strong in laboratory spectra 
are weak in the flare spectrum. An example is Fe $\operatorname{XIX}\left(2 s^{2} 2 p^{4}{ }^{1} D_{2}-2 s 2 p^{5}{ }^{1} P_{1}\right)$. This line is weak in the flare spectrum because the ${ }^{1} D_{2}$ level is not appreciably populated at flare electron densities.

Laboratory work on the $100 \AA$ lines is continuing, and needs to be extended through the beryllium iso-electronic sequence (Fe XXIII). The $100 \AA$ lines are particularly suitable for observations in solar flare spectra for two reasons: (a) there are few spectral lines from the quiet Sun near $100 \AA$, and (b) the lines are sufficiently wide at coronal flare temperatures $\left(\sim 10 \times 10^{6} \mathrm{~K}\right)$ to measure line profiles with existing high resolution grazing incidence spectrographs.

A byproduct of the line identifications near $100 \AA$ is the wavelength prediction of forbidden lines similar to the $5303 \AA$ line of Fe XIV. One of these lines $(1355 \pm 3 \AA$, Fe XXI $\left.\left({ }^{3} P_{0}-{ }^{3} P_{1}\right)\right)$ has already been found in the NRL Skylab data. These lines are particularly suitable for measurements of line widths and wavelength shifts.

\section{(c) SATELLITE LINES IN LABORATORY AND FLARE SPECTRA}

Most of the satellite lines identified so far in flare spectra are due to transitions in lithium-like and helium-like ions of the type, $1 s^{2} 2 l-1 s 2 p 2 l$, and $1 s 2 l-2 p 2 l$, respectively. Although some of the satellite lines were originally observed long ago in laboratory spectra by Edlén and Tyrén (1939), it was only recently that detailed classifications for the lines due to lithium-like ions were given by Gabriel and Jordan (1969a), Gabriel (1972) and Grineva et al. (1973). Classifications for the helium-like satellite lines of the heavier elements such as aluminum and silicon have recently been given by Feldman et al. (1974c). These authors have also observed and classified satellite lines in laboratory spectra near the $1 s^{2}{ }^{1} S_{0}-1 s 3 p{ }^{1} P_{1}$ helium-like lines due to transitions of the type, $1 s^{2} 2 l-1 s 2 l 3 p$. Satellite lines of this type are also observed in flare spectra (Neupert, 1971; Doschek and Meekins, 1973). Earlier work on helium-like satellites of the lighter elements is summarized in Walker and Rugge (1971), who identified helium-like satellites of magnesium in solar active region spectra.

Gabriel and Jordan (1969a) showed that the lithium-like satellite lines were formed by dielectronic recombination of the helium-like ion, and therefore the observation of these lines is a direct confirmation of the role of dielectronic recombination in shaping the ionization balance in the corona. Goldsmith (1969) had reached a similar conclusion concerning the formation of the helium-like satellites. Gabriel (1972) and Grineva et al. (1973) subsequently showed that the satellite lines could be used to determine the electron temperature of a plasma, and the departure of the plasma from ionization equilibrium. The impetus for solar and laboratory observations of the satellite lines arose in part from the identification by Gabriel and Jordan (1969a) of the forbidden line $\left(1 s^{2}{ }^{1} S_{0}-1 s 2 s^{3} S_{1}\right)$ of the helium-like ions in solar spectra of active regions and flares. The forbidden line may have diagnostic value for flares because the intensity ratio of the forbidden to intercombination line has been shown by Gabriel and Jordan (1969b) to be density sensitive.

Recent laboratory observations of satellite lines have been made using vacuum sparks (Schwob and Fraenkel, 1972: Elton and Lee, 1972; Lee, 1974; Margalit et al., 
1974; Holz et al., 1974), the plasma focus (Peacock et al., 1971), and laser-produced plasmas (Peacock et al., 1973; Feldman et al., 1974a, c). Satellite lines of iron have so far only been observed in vacuum spark spectra. Only the satellite lines of lithiumlike iron are strong in these spectra. Emission from Fe XXVI and associated satellite lines is quite weak.

Figure 7 shows a vacuum spark spectrum of iron obtained by Schwob and Fraenkel (1972), and Figure 8 shows a recently obtained solar flare iron spectrum reported by Grineva et al. (1973). (Recent results concerning high resolution solar satellite line spectra of magnesium and lighter elements are summarized in this Symposium by Parkinson (1975).) The spark spectra still do not have the resolution necessary to resolve the closely spaced lines near $1.86 \AA$. Furthermore, the solar and spark spectrum differ in that the solar spectrum is indicative of a plasma that is close to ionization equilibrium, while the spark spectrum indicates extreme transient ionization of the laboratory plasma (Gabriel, 1972). The presence of substantial innershell emission from the lower ionization stages such as Fe XVIII in the spark spectrum is indicative of extreme transient ionization. The forbidden line observed in the solar spectrum

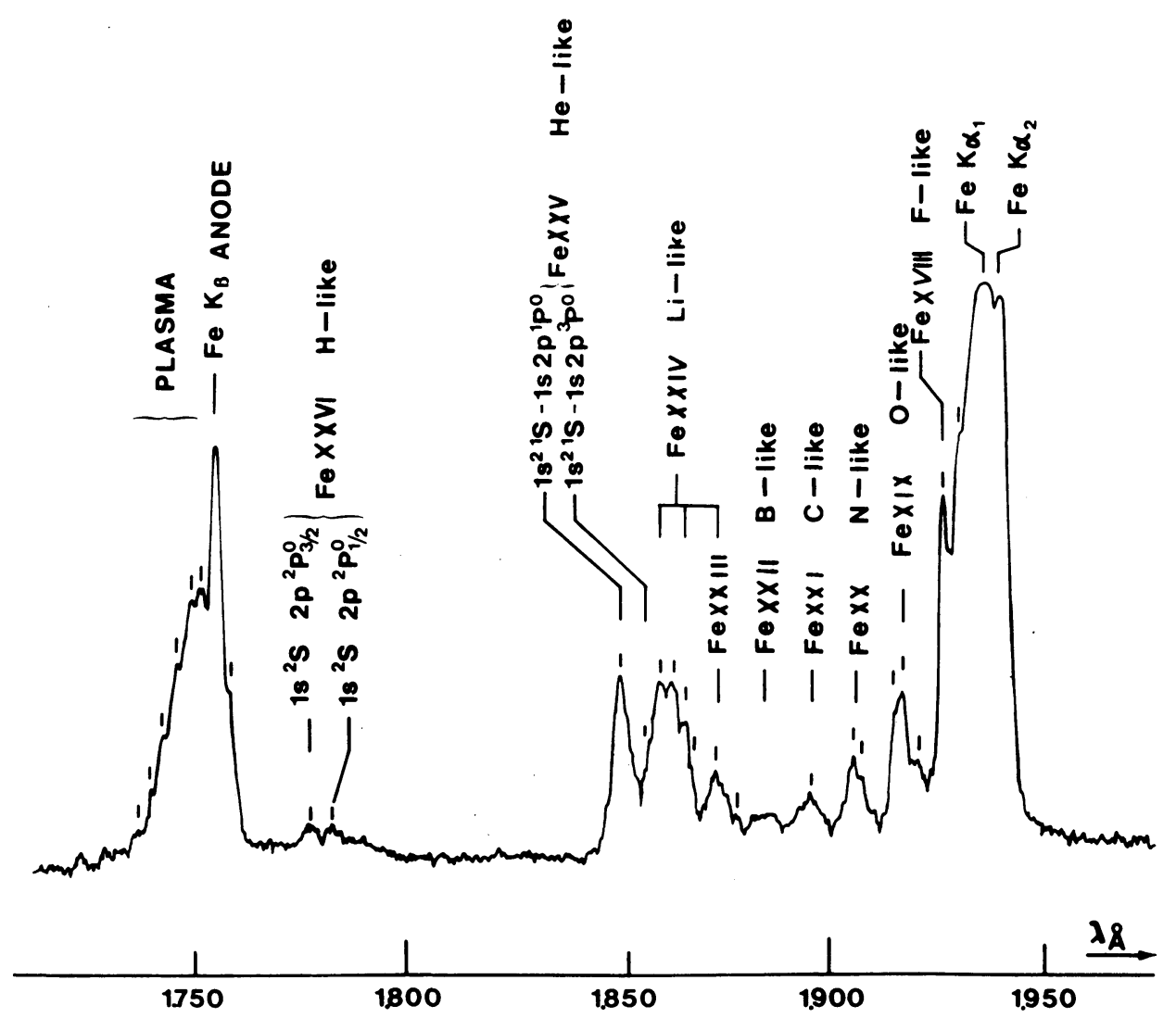

Fig. 7. A vacuum spark spectrum of iron. Many of the blended lines near $1.85 \AA$ are also prominent in solar flare spectra. (Spectrum courtesy of B. S. Fraenkel.) 


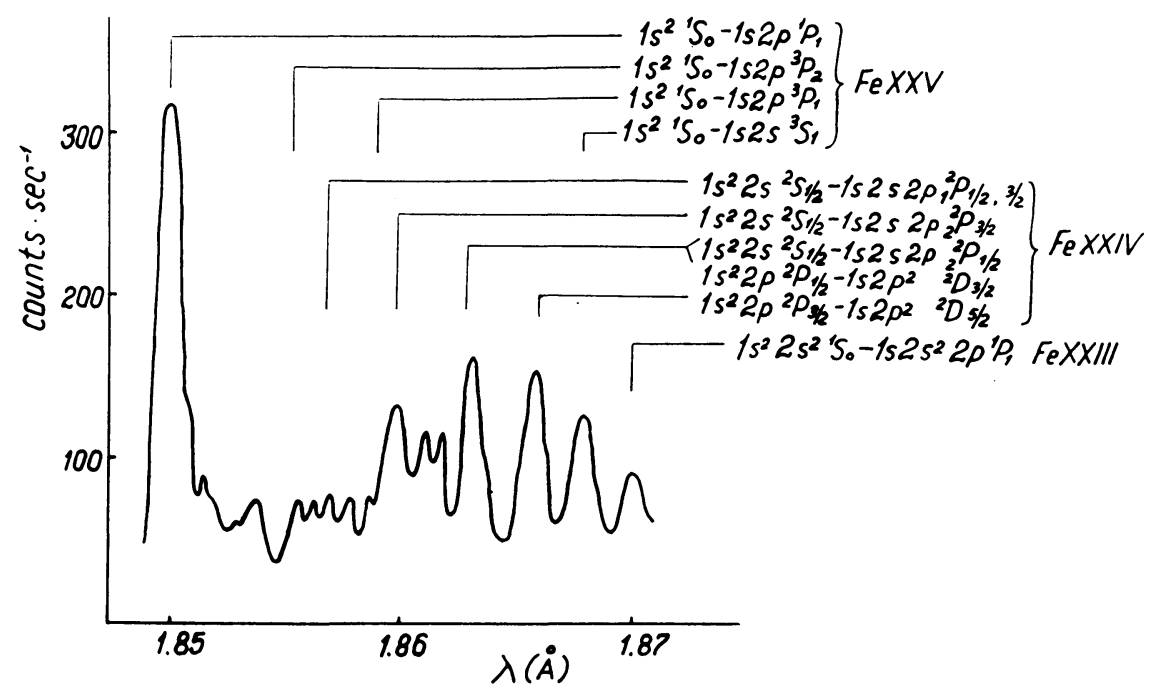

Fig. 8. Solar flare spectrum of iron obtained by Grineva et al. (1973). Most of the lines of Fe xxiv are produced by dielectronic recombination of Fe xxv. Compare with Figure 7.

TABLE I

Magnesium and calcium satellite lines ${ }^{\text {a }}$

\begin{tabular}{|c|c|c|c|}
\hline Key & Transition & $\lambda(\AA) \mathrm{Mg} \mathrm{x}$ & $\lambda(\AA)$ Ca xvIII \\
\hline $\mathrm{a}$ & $1 s^{2} 2 p^{2} P_{3 / 2}-1 s 2 p^{2}{ }^{2} P_{3 / 2}$ & 9.296 & 3.203 \\
\hline d & ${ }^{2} P_{1 / 2}-{ }^{2} P_{1 / 2}$ & 9.295 & 3.203 \\
\hline j & ${ }^{2} P_{3 / 2}$ & 9.321 & 3.210 \\
\hline $\mathrm{k}$ & ${ }^{2} P_{1 / 2}$ & 9.318 & 3.207 \\
\hline $\mathrm{m}$ & ${ }^{2} P_{3 / 2}-$ & 9.221 & 3.189 \\
\hline $\mathrm{n}$ & ${ }^{2} P_{1 / 2}-\quad{ }^{2} S_{1 / 2}$ & 9.218 & 3.185 \\
\hline $\mathrm{q}$ & $1 s^{2} 2 s^{2} S_{1 / 2}-1 s\left(2 s 2 p^{3} P\right)^{2} P_{3 / 2}$ & 9.283 & 3.200 \\
\hline $\mathrm{r}$ & ${ }^{2} S_{1 / 2} \quad{ }^{2} P_{1 / 2}$ & 9.286 & 3.202 \\
\hline s & ${ }^{2} S_{1 / 2}-1 s\left(2 s 2 p^{1} P\right)^{2} P_{3 / 2}$ & 9.235 & 3.191 \\
\hline $\mathrm{t}$ & ${ }^{2} S_{1 / 2}-{ }^{2} P_{1 / 2}$ & 9.236 & 3.192 \\
\hline $\mathrm{w}$ & $1 s^{2}{ }^{1} S_{0}-1 s 2 p^{1} P_{1}$ & 9.168 & 3.176 \\
\hline $\mathrm{y}$ & $1 s^{2}{ }^{1} S_{0}-1 s 2 p^{3} P_{1}$ & 9.231 & 3.192 \\
\hline
\end{tabular}

a Wavelengths calculated by Gabriel (1972).

shown in Figure 8 is not expected to contribute to the blend of emission in Figure 7, because of the much higher electron densities of spark plasmas.

Recently, satellite line spectra have been obtained from laser-produced plasmas. Figure 9 shows lithium-like satellite lines of magnesium obtained by Peacock $e t$ al. (1973). Figure 10 shows similar satellite spectra for heavier elements obtained by Feldman et al. (1974c). The letters over the lines and multiplets are the same key letters used by Gabriel (1972) in classifying the lines. Finally, Figure 11 shows heliumlike satellites of aluminum obtained by Feldman et al. (1974c). Also shown are satellite lines near the $1 s^{2}{ }^{1} S_{0}-1 s 3 p{ }^{1} P_{1}$ aluminum line. 
Table I gives Gabriel's (1972) theoretical wavelengths for lithium-like satellite lines of magnesium and calcium. The satellite line laser spectra are interesting because the relative intensities of the three pairs of lines, $(a, d),(q, r)$, and $(j, k)$ in Figures 9 and 10 are not in accord with theoretical expectations (Gabriel, 1972). However, similar anomalous intensity behavior is not observed in spectra obtained with vacuum sparks (Elton, 1974) or the plasma focus (Peacock et al., 1971), and therefore some aspect of the laser-produced plasmas may be responsible for the anomalous relative intensities. (See Note added in proof.)

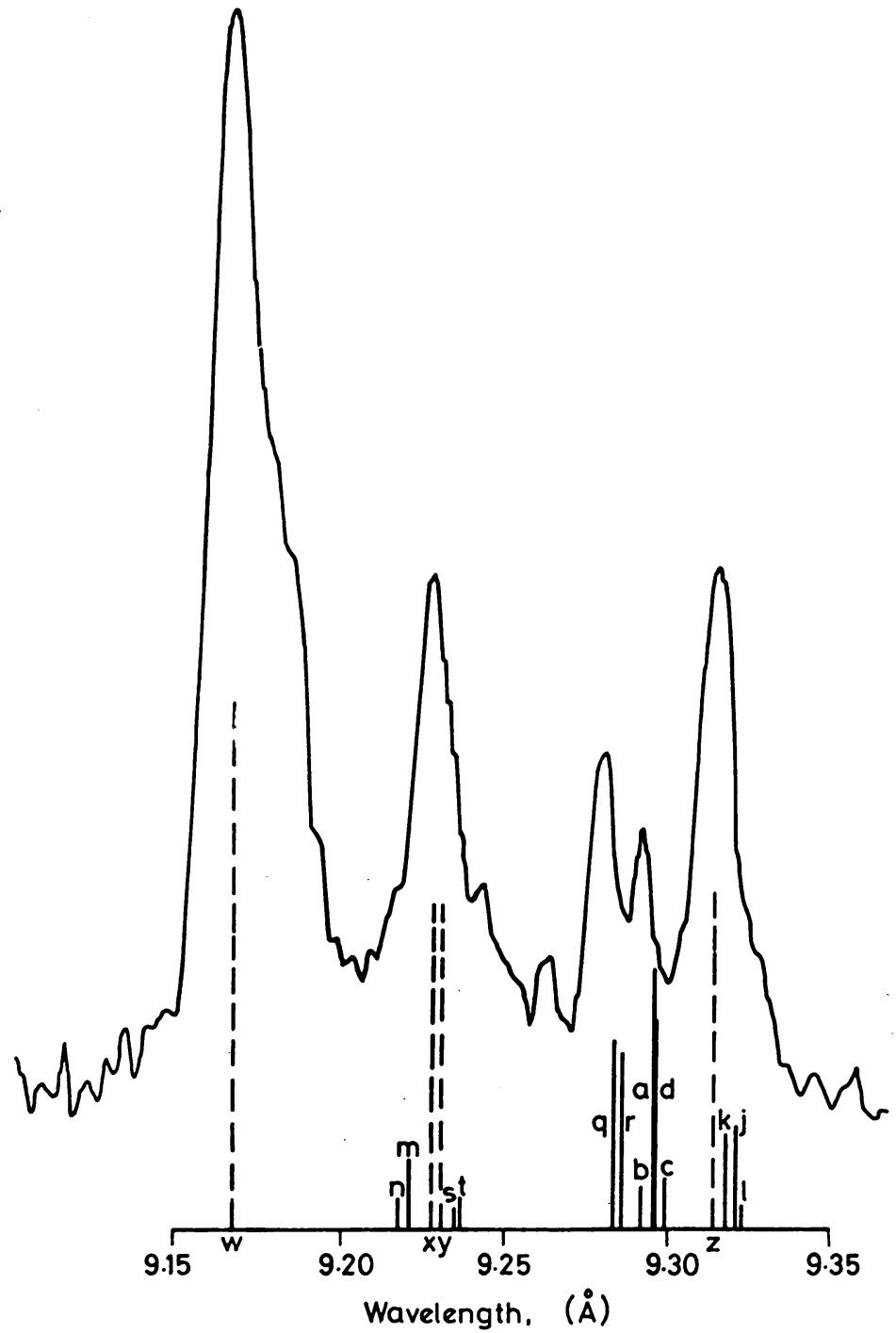

Fig. 9. Laser-produced plasma spectrum of helium-like and lithium-like magnesium. The line $z$ is the forbidden line $\left(1 s^{2}{ }^{1} S_{0}-1 s 2 s^{3} S_{1}\right)$ of $\mathrm{Mg}$ XI and may not be present in the laboratory spectrum because of the high electron density. The same remark may also apply to line $x$, a magnetic quadrupole transition $\left(1 s^{2}{ }^{1} S_{0} \cdots 1 s 2 p^{3} P_{2}\right)$. (Spectrum courtesy of N. J. Peacock.) 
Care must be exercised in interpreting laboratory spectra obtained photographically, because different types of lines can be formed at different times during the lifetime of the plasma and within different regions of the plasma. Peacock et al. (1973) report that the lithium-like satellite lines are formed close to the target surface in laser-produced

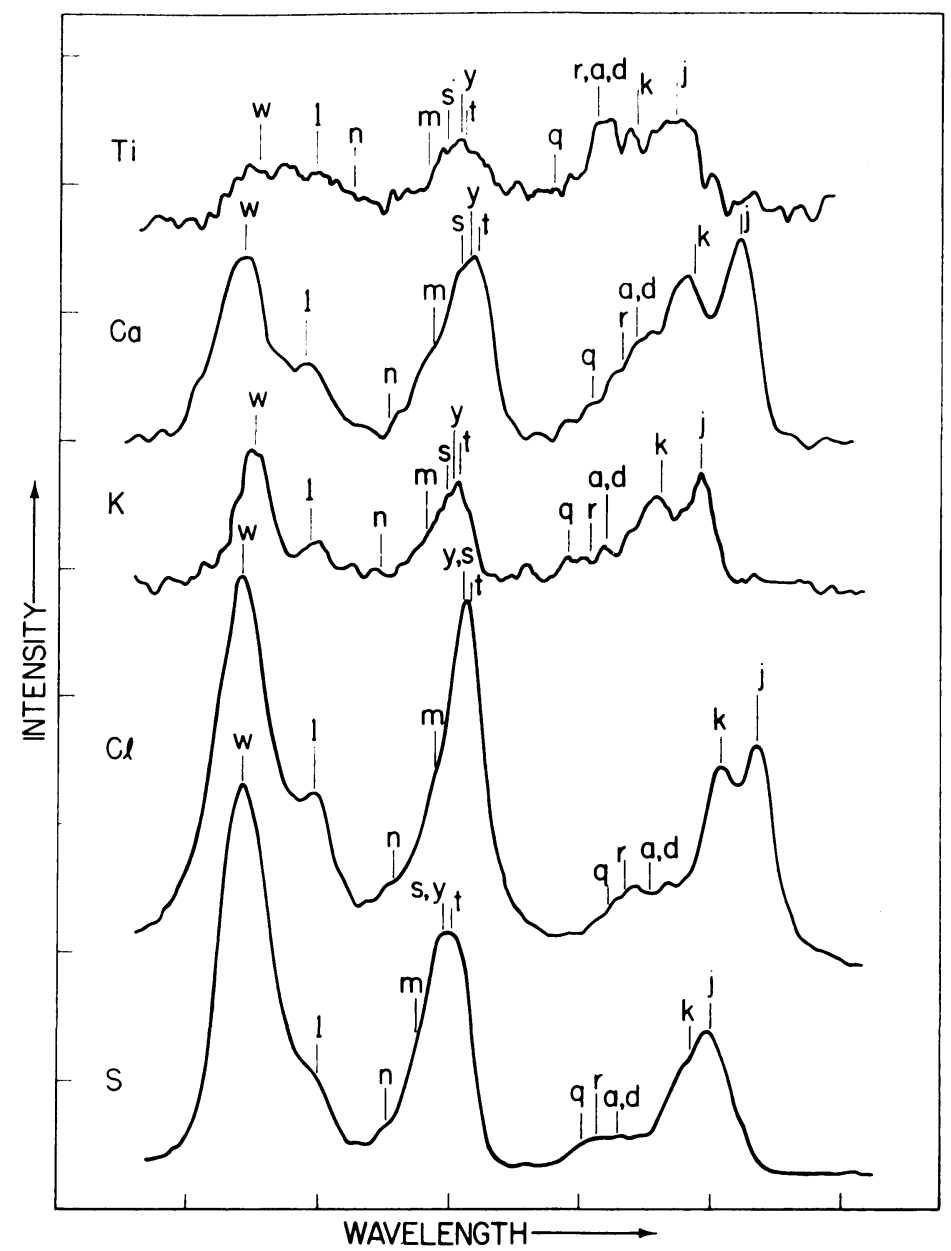

Fig. 10. Laser-produced plasma spectra of helium-like and lithium-like ions of heavier elements up through titanium (Compare with Figure 9.) In these laser-produced plasmas, satellite line emission for calcium and titanium is as strong or stronger than resonance line emission from the helium-like ions. This is not observed in solar flare spectra.

plasmas, while the resonance and intercombination lines are emitted in the plasma plume expanding away from the target. This is dramatically illustrated in a pinholetype spectrum of magnesium recently obtained by Burkhalter (1974), and shown in Figure 12. Emission from the resonance and intercombination lines can be seen as far as seven millimeters from the target surface. 


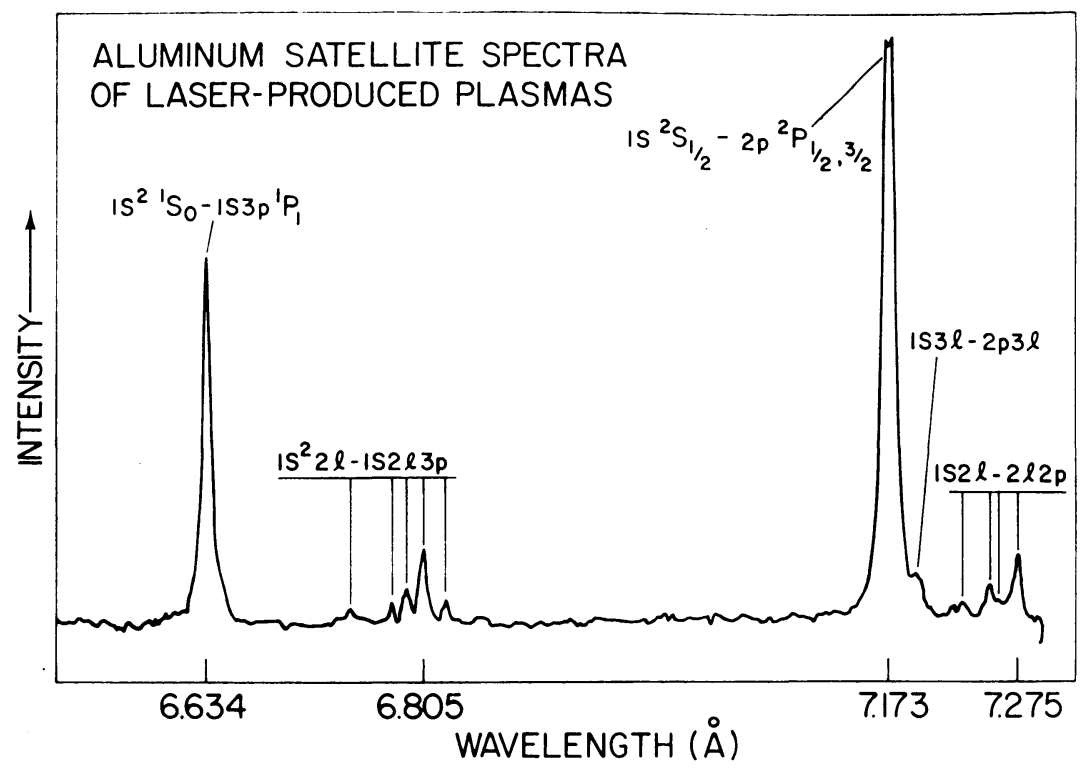

Fig. 11. Laser-produced plasma spectrum of satellite lines due to transitions of the type $1 s^{2} 2 l$ $-1 s 2 l 3 p$, and $1 s 2 l-2 p 2 l$. These types of satellite lines are also found in solar flare spectra.

\section{Mg TARGET}

\section{H - RESONANCE He-SATELLITE}

\section{IMPURITY}

\section{He - RESONANCE INTERCOMBINATION Li - SATELLITE}
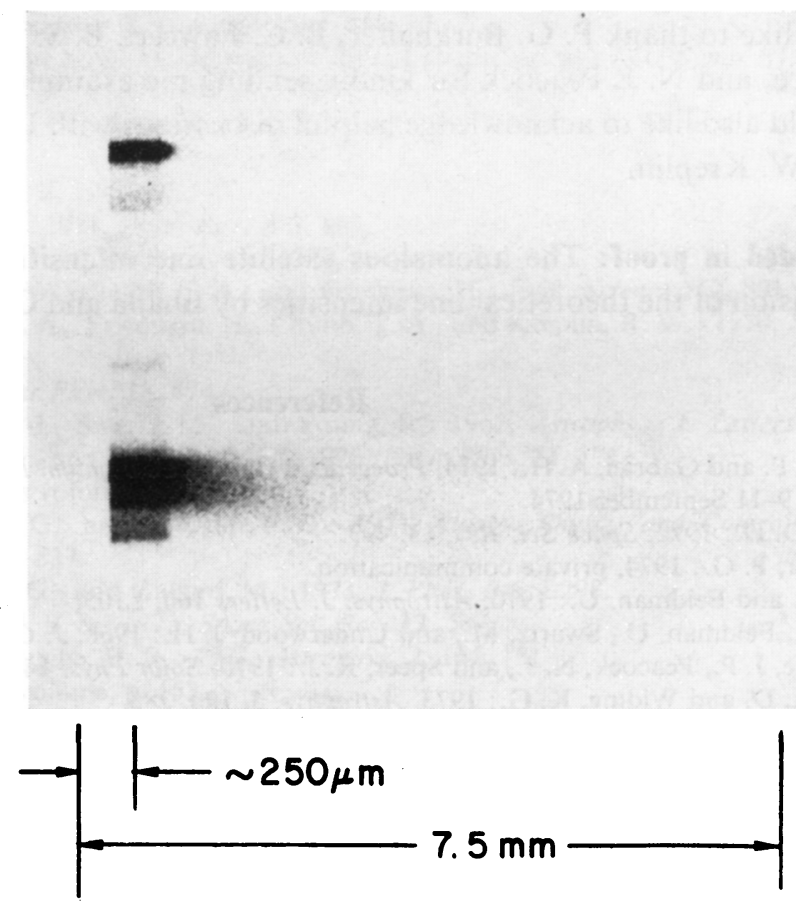

Fig. 12. Laser-produced plasma pinhole-type spectrum of hydrogen-like, helium-like, and lithiumlike magnesium obtained from a laser-produced plasma. Emission from satellite lines is confined near the target surface. Emission from the other lines is observed as much as $7 \mathrm{~mm}$ from the target surface.

Compare with Figure 9. (Spectrum courtesy of P. G. Burkhalter.) 
It is from the analysis of laboratory data such as in Figures 7-12 that the atomic physics in high temperature plasmas is clarified; and can therefore be applied with confidence to the analysis of astrophysical plasmas.

\section{Conclusions}

The recent classifications of iron lines in X-ray and EUV laboratory spectra, and recent $a b$ initio wavelength and oscillator strength predictions for iron lines in the $\mathrm{X}$-ray region, make possible new experiments in solar flare spectroscopy that enable the temperature-emission measure distribution of the plasma to be determined in flare plasmas. Line profiles can be measured for highly ionized iron lines in the $100 \AA$ region, and for similar lines in the $\mathrm{X}$-ray region.

From solar observations of satellite lines of the principal series lines of hydrogen-like and helium-like ions, electron temperatures and departures of flare plasmas from ionization equilibrium can be determined. The interpretation of the solar satellite line observations are made possible in part by analysis of spectra obtained from laboratory plasmas. In overall phenomenology, laboratory plasmas show a striking resemblance to solar flare plasmas.

\section{Acknowledgements}

I would like to thank P. G. Burkhalter, B. C. Fawcett, B. S. Fraenkel, S. O. Kastner, T. N. Lee, and N. J. Peacock for kindly sending me examples of their data.

I would also like to acknowledge helpful discussions with Uri Feldman, J. Meekins, and R. W. Kreplin.

Note added in proof: The anomalous satellite line intensities have been explained by a revision of the theoretical line intensities by Bhalla and Gabriel (1974).

\section{References}

Bhalla, C. P. and Gabriel, A. H.: 1974, Proceedings of IAU Colloquium No. 27, held at Harvard University, 9-11 September 1974.

Burgess, D. D.: 1972, Space Sci. Rev. 13, 493.

Burkhalter, P. G.: 1974, private communication.

Cohen, L. and Feldman, U.: 1970, Astrophys. J. Letters 160, L105.

Cohen, L., Feldman, U., Swartz, M., and Underwood, J. H.: 1968, J. Opt. Soc. Am. $58,843$.

Connerade, J. P., Peacock, N. J., and Speer, R. J.: 1970, Solar Phys. 14, 159.

Cowan, R. D. and Widing, K. G.: 1973, Astrophys. J. 180, 285.

Doschek, G. A.: 1972, Space Sci. Rev. 13, 765.

Doschek, G. A., Meekins, J. F., Kreplin, R. W., Chubb, T. A., and Friedman, H.: 1971, Astrophys. J. 170, 573.

Doschek, G. A., Meekins, J. F., and Cowan, R. D.: 1972, Astrophys. J. 177, 261.

Doschek, G. A., Meekins, J. F., and Cowan, R. D.: 1973, Solar Phys. 29, 125.

Doschek, G. A. and Meekins, J. F. : 1973, in R. Ramaty and R. G. Stone (eds.), High Energy Phenomena on the Sun, NASA SP-342, p. 262.

Doschek, G. A., Feldman, U., Cowan, R. D., and Cohen, Leonard: 1974a, Astrophys. J. $188,417$. Doschek, G. A., Feldman, U., and Cohen, L.: 1974b, J. Opt. Soc. Am., in press. 
Edlén, B. and Tyrén, F.: 1939, Nature 143, 940.

Elton, R. C.: 1974, private communication.

Elton, R. C. and Lee, T. N.: 1972, Space Sci. Rev. 13, 747.

Fawcett, B. C.: 1971, special publication, S.R.C. Astrophysics Research Unit, Culham Laboratory, England.

Fawcett, B. C. 1973, preprint: Review paper on The Identification of Emission Lines of Highly Ionized Atoms in the Solar Spectrum, to be published in Advances in Atomic and Molec. Physics.

Fawcett, B. C., Cowan, R. D., and Hayes, R. W.: 1974a, Astrophys. J. 187, 377.

Fawcett, B. C., Galanti, M., and Peacock, N. J.: 1974b, J. Phys. B 7, 1149.

Feldman, U. and Cohen, L.: 1968, Astrophys. J. Letters 151, L55.

Feldman, U., Doschek, G. A., Nagel, D. J., Behring, W. E., and Cohen, Leonard: 1973a, Astrophys. J. Letters 183, L43.

Feldman, U., Doschek, G. A., Cowan, R. D., and Cohen, Leonard: 1973b, J. Opt. Soc. Am. 63, 1445.

Feldman, U., Doschek, G. A., Nagel, D. J., Behring, W. E., and Cowan, R. D.: 1974a, Astrophys. J. $187,417$.

Feldman, U., Doschek, G. A., Cowan, R. D., and Cohen, Leonard: 1974b, Astrophys. J., in press.

Feldman, U., Doschek, G. A., Nagel, D. J., Cowan, R. D., and Whitlock, R. R.: 1974c, Astrophys. J. 192, 213.

Gabriel, A. H.: 1972, Monthly Notices Roy. Astron. Soc. 160, 99.

Gabriel, A. H. and Jordan, C.: 1969a, Nature 221, 947.

Gabriel, A. H. and Jordan, C.: 1969b, Monthly Notices Roy. Astron. Soc. 145, 241.

Goldsmith, S.: 1969, J. Phys. B 2, 1075.

Grineva, Yu. I., Karev, V. I., Korneev, V. V., Krutov, V. V., Mandelstam, S. L., Vainstein, L. A., Vasilyev, D. N., and Zhitnik, I. A.: 1973, Solar Phys. 29, 441.

Holz, E. Ya., Kononov, E. Ya., Mandelstam, S. L., Sidel'nikov, Yu. V., Zitnik, I. A.: 1974, paper presented at the XVII Plenary Meeting of COSPAR, June-July 1974, Sao Paulo, Brazil.

Jordan, C.: 1969, Monthly Notices Roy. Astron. Soc. 142, 501.

Jordan, C.: 1970, Monthly Notices Roy. Astron. Soc. 148, 17.

Kane, S. R.: 1974, in Gordon Newkirk, Jr. (ed.), 'Coronal Disturbances', IAU Symp. 57, 105.

Kastner, S. O., Neupert, W. M., and Swartz, M.: 1974, Astrophys. J. 191, 261.

Krieger, A. S., Chase, R. C., Gerassimenko, M., Kahler, S. W., Timothy, A. F., and Vaiana, G. S.: 1975 , This volume, p. 103.

Lee, T. N.: 1974, Astrophys. J. 190; 467.

Lee, T. N. and Elton, R. C.: 1971, Phys. Rev. A 3, 865.

Margalit, G., Goldsmith, S., and Feldman, U.: 1974, preprint.

Meekins, J. F., Kreplin, R. W., Chubb, T. A., and Friedman, H.: 1968, Science 162, 891.

Meekins, J. F., Doschek, G. A., Friedman, H., Chubb, T. A., and Kreplin, R. W. : 1970, Solar Phys. $13,198$.

Neupert, W. M.: 1971, Solar Phys. 18, 474.

Neupert, W. M., Gates, W. J., Swartz, M., and Young, R.: 1967, Astrophys. J. Letters 149, L79.

Neupert, W. M., Swartz, M., and Kastner, S. O.: 1973, Solar Phys. 31, 171.

Parkinson, J. H.: 1975, This volume, p. 45.

Peacock, N. J., Hobby, M. G., and Morgan, P. D.: 1971, Plasma Physics and Controlled Fusion, Vol. 1, IAEA, Vienna, p. 537.

Peacock, N. J., Hobby, M. G., and Galanti, M.: 1973, J. Phys. B 6, L298.

Schwob, J. L. and Fraenkel, B. S.: 1972, Space Sci. Rev. 13, 589.

Walker, A. B. C., Jr. and Rugge, H. R.: 1971, Astrophys. J. 164, 181.

Widing, K. G.: 1975, This volume, p. 153. 